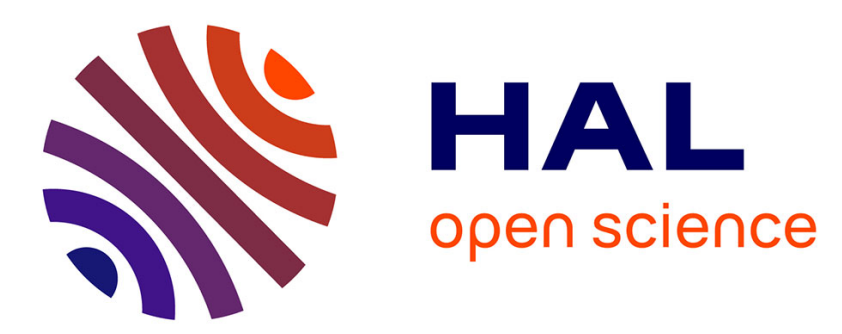

\title{
Bounded attitude control of rigid bodies: Real-time experimentation to a quadrotor mini-helicopter
}

Fermi Guerrero-Castellanos, Nicolas Marchand, Ahmad Hably, Suzanne Lesecq, Jérôme Delamare

\section{- To cite this version:}

Fermi Guerrero-Castellanos, Nicolas Marchand, Ahmad Hably, Suzanne Lesecq, Jérôme Delamare. Bounded attitude control of rigid bodies: Real-time experimentation to a quadrotor mini-helicopter. Control Engineering Practice, 2011, 19 (8), pp.790-797. 10.1016/j.conengprac.2011.04.004 . hal00568075

\section{HAL Id: hal-00568075 \\ https://hal.science/hal-00568075}

Submitted on 4 Jul 2015

HAL is a multi-disciplinary open access archive for the deposit and dissemination of scientific research documents, whether they are published or not. The documents may come from teaching and research institutions in France or abroad, or from public or private research centers.
L'archive ouverte pluridisciplinaire HAL, est destinée au dépôt et à la diffusion de documents scientifiques de niveau recherche, publiés ou non, émanant des établissements d'enseignement et de recherche français ou étrangers, des laboratoires publics ou privés. 


\title{
Bounded attitude control of rigid bodies: Real-time experimentation to a quadrotor mini-helicopter
}

\author{
J.F. Guerrero-Castellanos ${ }^{\mathrm{a}}$, N. Marchand ${ }^{\mathrm{b}}$, A. Hably ${ }^{\mathrm{b}, *}$, \\ S. Lesecq ${ }^{\mathrm{c}}$, J. Delamare ${ }^{\mathrm{d}}$ \\ ${ }^{a}$ Autonomous University of Puebla (BUAP), Faculty of Electronics Av. San Claudio y \\ 18 Sur, Ciudad Universitaria 72570, Puebla, Mexico \\ ${ }^{b}$ GIPSA-lab - Control Systems department - ENSE3 bat B - BP 46, Domaine \\ Universitaire, 38400 Saint Martin d'Heres, France \\ ${ }^{c}$ CEA DRT/LETI/DCIS/PILSI, 17 rue des martyrs, 38054 Grenoble, France \\ ${ }^{d}$ G2Elab - ENSE3 bat D - BP 46, Domaine Universitaire, 38400 St Martin d'Heres \\ Cedex, France
}

\begin{abstract}
A quaternion-based feedback is developed for the attitude stabilization of rigid bodies. The control design takes into account a priori input bounds and is based on nested saturation approach. It results in a very simple controller suitable for an embedded use with low computational resources available. The proposed method is generic not restricted to symmetric rigid bodies and does not require the knowledge of the inertia matrix of the body. The control law can be tuned to force closed-loop trajectories to enter in some a priori fixed neighborhood of the origin in a finite time and remain thereafter. The global stability is guaranteed in the case where angular velocities sensors have limited measurement range. The control law is experimentally applied to the attitude stabilization of a quadrotor mini-helicopter.
\end{abstract}

Keywords: Nonlinear control, attitude stabilization, embedded control,

\footnotetext{
${ }^{*}$ Corresponding author, fax: 0033476826388

Email address: ahmad.hably@gipsa-lab.grenoble-inp.fr (A. Hably)
} 
quadrotor helicopter

\section{Introduction}

The problem of attitude control of a rigid body has attracted considerable amount of interest since the 1950's within the scientific communities of aeronautics, aerospace, control and robotics. Indeed many systems such as spacecrafts, satellites, helicopters, tactical missiles, coordinated robot manipulators, underwater vehicles, aerial vehicles and others can enter within the framework of rigid bodies with a need for attitude control. Several approaches were applied such as feedback linearizing control law (Wie et al. (1989); Fjellstad and Fossen (1994)), feedback proportional-derivative control law (Wen and Kreutz-Delgado (1991); Egeland and Godhavn (1994); Tsiotras (1994); Joshi et al. (1995)), predictive control (applied to a spacecraft in Wen et al. (1997) and a to micro-satellite in Hegrenas et al. (2005)), backstepping (quaternion based in Kristiansen and Nicklasson (2005) and nonlinear adaptive in Singh and Yim (2002)), robust control applied to tactical missiles (Song et al. (2005)). This list is of course far from being exhaustive. Within these mentioned approaches, a feedback linearization coupled with a proportional-derivative control is probably the most widely used method to solve the attitude control problem. This ensures stabilization with a simple implementation of the control law. Sometimes, the linearization step is even not applied. The major criticism of this approach is that for large attitude or angular velocity errors a large control effort is required. Furthermore, the linearization step requires a relatively accurate model of the system.

In practice, the limitations on available energy impose bounded input signal. 
Moreover, it is common that the output of the system are bounded due to sensors limitation. Actually, the above cited attitude control approaches do not consider the problem which takes the input and/or output constraints into account. Few publications have dealt with this problem. In Tsiotras and Luo (2000), the stabilization of an underactuated rigid spacecraft subject to input constraints is studied. Although this approach uses an innovative attitude representation that allows the decomposition of general motion into two rotations, the proposed control law and its analysis are restricted only to the kinematic level. In Belta (2004), a control law that drives a rigid underwater vehicle between arbitrary initial and final region of the state space while satisfying bounds on control and state is proposed. The approach is based in a control of multi-affine systems. The authors in Boskovic et al. (1999) have studied the robust sliding mode stabilization of the spacecraft attitude dynamics in presence of control input saturation based on the variable structure control (VSC) approach. Unfortunately, the stabilizing bounded control laws applied in these works are non smooth and this fact renders difficult their practical implementation. The application of optimal control of a rigid body's attitude has been the interest of many researches (see Scrivener and Thompson (1994) and references therein). However, when the problem is subject to control constraints a difficulty appears. Actually, one must solve the Hamilton-Jacobi equation and thus computing optimal control is generally infeasible for operational attitude control systems. Different works have circumvented this difficulty using the so-called inverse optimal method (Osipchuck et al. (1997); Krstić and Tsiotras (1999)). In this approach, the knowledge of a Control Lyapunov Function (CLF) and a stabilizing control 
law is required. The authors in Krstić and Tsiotras (1999) exploit the cascaded interconnection structure of the dynamics and kinematics equations of the rigid body to construct a control law based on backstepping. However, the optimal control law depends on the moment of inertia and the robustness issues with respect to model's errors are not addressed. In Osipchuck et al. (1997), a CLF and a corresponding stabilizing control solution of a static quadratic programming problem are proposed. Unfortunately, the control law here is again not smooth. More recently, an elegant quaternion based output feedback for the attitude tracking problem is proposed in Tayebi (2008). In the regulation case, the proposed control law is structurally bounded. However, the control gains are restricted to be identical for each axis. Furthermore, this approach does not take into consideration the angular velocity constraints.

The approach proposed in the present paper is more in the spirit of Wie and $\mathrm{Lu}$ (1995) where the problem of reorienting a rigid spacecraft within the physical limits of actuators has been investigated based on the nested saturation approach proposed by Teel (1992) for linear chain of integrators. However, in Wie and Lu (1995) no formal stability proof is given and only rest-to-rest maneuvers are investigated. The orientation of a rigid body can be parameterized by several ways (Shuster (1993)). As detailed in Section 2 , the unit quaternion attitude representation is adopted. This representation is a four-parameter global nonsingular parametrization of the attitude (Chou (1992)). This section also gives the corresponding equation of motion of rigid bodies and formulates the related stabilization control problem addressed in this paper. Theorem 1 in Section 3 is the main contribution of 
this paper. It presents a smooth nonlinear bounded control law that almost globally asymptotically stabilizes rigid bodies. A discontinuous version of the control law is also given in Remark 1. It circumvents the topological obstruction of global continuous stabilization in $S O(3)$. The results are based on the usage of nested saturation functions and can be seen, in a certain way, as a bounded PD controller. PD controller is already known to be suitable for attitude stabilization. In Bang et al. (2003), the unsaturated PD controller takes an analog form as the unsaturated form of the one proposed in this paper. However, in Bang et al. (2003) the stability of the bounded PD controller obtained with an anti-windup scheme is not proved. With the proposed control approach, the almost global stability can formally be established and, in addition, one can prove that the stability is not affected neither by a bad knowledge of the inertia matrix (actually, it is even not necessary to know it) nor by slew rate limits (presented by all real angular velocity sensors). Finally, in Section 4 the result is applied to a quadrotor mini-helicopter. This system exhibits in addition to rigid bodies, gyroscopic torques generated by the rotating propellers. It is formally established that these terms do not affect the almost global asymptotic stability of the control law proposed in Section 3.

\section{Mathematical Background and problem statement}

\subsection{Quaternion formalism and rigid body dynamics}

The attitude of a rigid body can be represented by a quaternion, consisting of a unit vector $\vec{e}$, known as the Euler axis, and a rotation angle $\beta$ about 
this axis. The quaternion $q$ is then defined as follows:

$$
q=\left[\begin{array}{c}
\cos \frac{\beta}{2} \\
\vec{e} \sin \frac{\beta}{2}
\end{array}\right]=\left[\begin{array}{c}
q_{0} \\
\vec{q}
\end{array}\right] \in \mathbb{H}
$$

where

$$
\mathbb{H}=\left\{q \mid q_{0}^{2}+\vec{q}^{T} \vec{q}=1, q=\left[\begin{array}{ll}
q_{0} & \vec{q}
\end{array}\right]^{T}, q_{0} \in \mathbb{R}, \vec{q} \in \mathbb{R}^{3}\right\}
$$

$\vec{q}=\left[\begin{array}{lll}q_{1} & q_{2} & q_{3}\end{array}\right]^{T}$ and $q_{0}$ are known as the vector and scalar parts of the quaternion respectively. In attitude control applications, the unit quaternion represents the rotation from an inertial coordinate system $N\left(x_{n}, y_{n}, z_{n}\right)$ located at some point of the space (for instance, the earth NED frame), to the body coordinate system $B\left(x_{b}, y_{b}, z_{b}\right)$ located at the center of mass of the rigid body.

The rotation matrix $C(q)$ corresponding to the attitude quaternion $q$ is computed as:

$$
C(q)=\left(q_{0}^{2}-\vec{q}^{T} \vec{q}\right) I_{3}+2\left(\vec{q} \vec{q}^{T}-q_{0}\left[\vec{q}^{\times}\right]\right)
$$

where $I_{3}$ is the identity matrix and $\left[\xi^{\times}\right]$is the skew symmetric tensor associated with the axial vector $\xi$

$$
\left[\xi^{\times}\right]=\left[\begin{array}{l}
\xi_{1} \\
\xi_{2} \\
\xi_{3}
\end{array}\right]^{\times}=\left[\begin{array}{ccc}
0 & -\xi_{3} & \xi_{2} \\
\xi_{3} & 0 & -\xi_{1} \\
-\xi_{2} & \xi_{1} & 0
\end{array}\right]
$$

Denoting by $\vec{\omega}=\left[\begin{array}{lll}\omega_{1} & \omega_{2} & \omega_{3}\end{array}\right]^{T}$ the angular velocity vector of the body frame $B$ relative to the inertial frame $N$, expressed in $B$, the kinematics equation is given by:

$$
\left[\begin{array}{c}
\dot{q}_{0} \\
\dot{\vec{q}}
\end{array}\right]=\frac{1}{2}\left[\begin{array}{c}
-\vec{q}^{T} \\
I_{3} q_{0}+\vec{q}^{\times}
\end{array}\right] \vec{\omega}
$$




$$
=\frac{1}{2} \Xi(q) \vec{\omega}
$$

The attitude dynamics for a rigid body is described by:

$$
J \dot{\vec{\omega}}=-\vec{\omega} \times J \vec{\omega}+\Gamma
$$

where $J \in \mathbb{R}^{3 \times 3}$ is the symmetric positive definite constant inertial matrix of the rigid body expressed in the $B$ frame and $\Gamma \in \mathbb{R}^{3}$ is the vector of applied torques. These torques depend on the couples generated by the actuators, aerodynamic couples such as gyroscopic couples, gravity gradient, etc. In this paper, it is assumed that the body-fixed control axes coincide with the principal axes of inertia, so the inertia matrix is diagonal.

The attitude error is used to quantify the mismatch between two attitudes. If $q$ defines the current attitude quaternion and $q_{d}$ is the reference quaternion, i.e. the desired orientation, then the error quaternion that represents the attitude error between the current orientation and the desired one is given by:

$$
q_{e}=q \otimes q_{d}^{-1}
$$

where $\otimes$ denotes the quaternion multiplication and $q^{-1}$ is the complementary rotation of the quaternion $q$ which is the quaternion conjugate (Shuster (1993)).

\subsection{Problem Statement}

The objective is to design a control law that drives the rigid body attitude to a specified constant orientation and maintains this orientation starting from any initial condition. It follows that the angular velocity vector must be brought to zero and remains null. Let $q_{d}$ denote the desired constant rigid 
body orientation, the control objective is then described by the following asymptotic condition:

$$
q \rightarrow q_{d}, \quad \vec{\omega} \rightarrow 0 \text { as } t \rightarrow \infty
$$

If $q_{d}$ denotes the desired constant rigid body orientation, then the error quaternion that represents the attitude error between the current orientation and the desired one is given by equation (7). If the inertial coordinate frame is selected to be the desired orientation then the desired quaternion becomes $q_{d}=\left[\begin{array}{llll} \pm 1 & 0 & 0 & 0\end{array}\right]^{T}$ and the error quaternion (7) coincides with the current attitude quaternion, that is, $q_{e}=q$. This control objective is then:

$$
q \rightarrow\left[\begin{array}{llll} 
\pm 1 & 0 & 0 & 0
\end{array}\right]^{T}, \quad \vec{\omega} \rightarrow 0 \quad \text { as } t \rightarrow \infty
$$

Equation (9) represents two equilibrium points $\left(q_{0}=1, \vec{q}=\left[\begin{array}{lll}0 & 0 & 0\end{array}\right]^{T}\right)$ and $\left(q_{0}=-1, \vec{q}=\left[\begin{array}{lll}0 & 0 & 0\end{array}\right]^{T}\right)$. These equilibrium points represent the same equilibrium point in the physical space and they yield the same attitude matrix in equation (3). However, they represent two-point set in the quaternion space. This topological obstruction does not allow to state any global property for the closed-loop system, using a continuous quaternion-based feedback (Bhat and Bernstein (1998)). In this study, first the case $q_{d}=\left[\begin{array}{llll}1 & 0 & 0 & 0\end{array}\right]^{T}$ is considered in order to guarantee almost global closed-loop stability (Koditschek (1988)) using a continuous control law. Afterwards, global asymptotic stability will be claimed and the results will be generalized to any desired orientation.

The presence of actuator amplitude limitations can lead to undesirable closedloop behaviors even instability. Consequently, in addition to asymptotic stability, the feedback control must explicitly take into account the physical 
constraints in order to avoid unwanted damages and to maximize its effectiveness. Therefore, the control torques $\Gamma$ are required to be such as:

$$
\Gamma_{i} \in\left[-\bar{\Gamma}_{i}, \bar{\Gamma}_{i}\right] \text { with } i \in\{1,2,3\}
$$

where $\bar{\Gamma}_{i}$ represents the bound of the control torque $\Gamma_{i}$.

\section{Bounded Attitude Control}

\subsection{Control law formulation}

In this section, a control law that stabilizes the system described by (5) and (6) is proposed. The goal is to design a control torque that is bounded. This is achieved with the usage of the saturation function $\sigma_{M}$ defined in the Definition 1.

Definition 1 (Saturation function). Let $\sigma_{M}: \mathbb{R} \rightarrow \mathbb{R}$ denote the classical saturation function defined by :

1. $\sigma_{M}(s)=s$ if $|s|<M$;

2. $\sigma_{M}(s)=\operatorname{sign}(s) M$ elsewhere;

With the above definition, our main result is the following:

Theorem 1. Consider the rigid body rotational dynamics described by (5) and (6) with the following bounded control inputs $\Gamma=\left[\begin{array}{lll}\Gamma_{1} & \Gamma_{2} & \Gamma_{3}\end{array}\right]^{T}$ defined by:

$$
\Gamma_{i}=-\sigma_{\bar{\Gamma}_{i}}\left(\frac{\kappa \omega_{i}}{\rho_{i}}+\kappa q_{i}\right)
$$

$\sigma(\cdot)$ are saturation functions as defined above. $\bar{\Gamma}_{i}$ with $i \in\{1,2,3\}$ represents the physical bound on the $i^{\text {th }}$ torque $\Gamma_{i} . \kappa$ is a real parameter such that $0<$ 
$\kappa \leq \min _{i} \bar{\Gamma}_{i} / 2 . \rho_{i}$ are strictly positive real parameters. Then the inputs (11) almost globally asymptotically stabilize the rigid body to the origin $\left(q_{0}=\right.$ $1, \vec{q}=0$ and $\vec{\omega}=0)$.

Proof. Consider first the the Lyapunov function:

$$
V=\frac{1}{2} \vec{\omega}^{T} J \vec{\omega}
$$

Then, along the trajectories of the system, one has:

$$
\dot{V}=\underbrace{\vec{\omega}^{T}(-\vec{\omega} \times J \vec{\omega})}_{=0}+\vec{\omega}^{T} \Gamma=\underbrace{\omega_{1} \Gamma_{1}}_{\dot{V}_{1}}+\underbrace{\omega_{2} \Gamma_{2}}_{\dot{V}_{2}}+\underbrace{\omega_{3} \Gamma_{3}}_{\dot{V}_{3}}
$$

$\dot{V}$ is the sum of three terms $\dot{V}_{1}, \dot{V}_{2}$ and $\dot{V}_{3}$. Analyzing $\dot{V}_{i}$ for $i \in\{1,2,3\}$, one gets from $\Gamma_{i}$ in (11) and equation (13):

$$
\dot{V}_{i}=-\omega_{i} \sigma_{\bar{\Gamma}_{i}}\left(\frac{\kappa \omega_{i}}{\rho_{i}}+\kappa q_{i}\right)
$$

Let $\Phi_{i}:=\left\{\omega_{i}:\left|\omega_{i}\right| \leq \rho_{i}+\varepsilon\right\}$ for some small $\varepsilon>0$. Outside $\Phi_{i}$, from the quaternion's condition $\left|q_{i}\right| \leq 1$, it follows that $\left|\frac{\kappa \omega_{i}}{\rho_{i}}+\kappa q_{i}\right| \geq \frac{\kappa \varepsilon}{\rho_{i}}$ and that $\frac{\kappa \omega_{i}}{\rho_{i}}+\kappa q_{i}$ and $\omega_{i}$ have the same sign. Therefore:

$$
\dot{V}_{i}=-\omega_{i} \sigma_{\bar{\Gamma}_{i}}\left(\frac{\kappa \omega_{i}}{\rho_{i}}+\kappa q_{i}\right) \leq-\frac{\kappa \varepsilon}{\rho_{i}}\left|\omega_{i}\right|<-\frac{\kappa \varepsilon\left(\rho_{i}+\varepsilon\right)}{\rho_{i}}<-\kappa \varepsilon<0
$$

Consequently, $\omega_{i}$ enters $\Phi_{i}$ in a finite time and remains in it thereafter. During that time, the quaternion cannot diverge since it is structurally unitary and therefore bounded..

Once in $\Phi_{i}$, let us consider the new Lyapunov function $W$ defined by:

$$
\begin{aligned}
W & =\frac{1}{2} \vec{\omega}^{T} J \vec{\omega}+\kappa\left(\left(1-q_{0}\right)^{2}+\vec{q}^{T} \vec{q}\right) \\
& =\frac{1}{2} \vec{\omega}^{T} J \vec{\omega}+2 \kappa\left(1-q_{0}\right)
\end{aligned}
$$


Since $J$ is positive definite, the function $W$ is positive definite, radially unbounded and is of class $C^{2}$. The derivative of (16) after using (5) and (6) is given by:

$$
\begin{aligned}
\dot{W} & =\vec{\omega}^{T} J \dot{\vec{\omega}}-2 \kappa \dot{q}_{0} \\
& =\underbrace{\vec{\omega}^{T} J(-\vec{\omega} \times J \vec{\omega})}_{=0}+\vec{\omega}^{T} \Gamma+\kappa \vec{q}^{T} \vec{\omega} \\
& =\vec{\omega}^{T} \Gamma+\kappa \vec{q}^{T} \vec{\omega} \\
& =\underbrace{\omega_{1} \Gamma_{1}+\kappa q_{1} \omega_{1}}_{\mathscr{W}_{1}}+\underbrace{\omega_{2} \Gamma_{2}+\kappa q_{2} \omega_{2}}_{\mathscr{W}_{2}}+\underbrace{\omega_{3} \Gamma_{3}+\kappa q_{3} \omega_{3}}_{\mathscr{W}_{3}}
\end{aligned}
$$

As for $V, \dot{W}$ is the sum of three terms $\dot{W}_{1}, \dot{W}_{2}$ and $\dot{W}_{3}$. Analyzing $\dot{W}_{i}$ for $i \in\{1,2,3\}$, one gets from $\Gamma_{i}$ in (11) and equation (13):

$$
\dot{W}_{i}=-\omega_{i} \sigma_{\bar{\Gamma}_{i}}\left(\frac{\kappa \omega_{i}}{\rho_{i}}+\kappa q_{i}\right)+\kappa q_{i} \omega_{i}
$$

In $\Phi_{i}$, one has:

$$
\left|\frac{\kappa \omega_{i}}{\rho_{i}}+\kappa q_{i}\right| \leq 2 \kappa+\frac{\kappa \varepsilon}{\rho_{i}}
$$

Taking $\varepsilon$ sufficiently small und using the assumption that $2 \kappa<\bar{\Gamma}_{i}$,

$$
\left|\frac{\kappa \omega_{i}}{\rho_{i}}+\kappa q_{i}\right| \leq \bar{\Gamma}_{i}
$$

Consequently, $\sigma$ operates in a linear region:

$$
\Gamma_{i}=-\frac{\kappa}{\rho_{i}}\left[\omega_{i}+\rho_{i} q_{i}\right]
$$

As a result, (18) becomes:

$$
\dot{W}_{i}=-\frac{\kappa}{\rho_{i}} \omega_{i}^{2}
$$

Therefore, (17) becomes

$$
\begin{aligned}
\dot{W} & =\dot{W}_{1}+\dot{W}_{2}+\dot{W}_{3} \\
& =-\kappa\left(\frac{\omega_{1}^{2}}{\rho_{1}}+\frac{\omega_{2}^{2}}{\rho_{2}}+\frac{\omega_{3}^{2}}{\rho_{3}}\right) \leq 0
\end{aligned}
$$


In order to complete the proof, the LaSalle Invariance Principle is invoked. All the trajectories converge to the largest invariant set $\bar{\Omega}$ in $\Omega$

$$
\Omega=\{(\vec{q}, \vec{\omega}): \dot{W}=0\}=\{(\vec{q}, \vec{\omega}): \vec{\omega}=0\}
$$

In the invariant set, $J \dot{\vec{\omega}}=-\kappa\left[\begin{array}{lll}q_{1} & q_{2} & q_{3}\end{array}\right]^{T}=-\kappa \vec{q}=0$. Therefore to remain in $\bar{\Omega}$, one must satisfy $\vec{q}=0$ and $q_{0}= \pm 1$ from the normality condition. Actually, the points $\left(q_{0}= \pm 1, \vec{q}=0, \vec{\omega}=0\right)$ correspond, respectively, to a minimum $(W=0)$ and a local maximum $(W=4)$ of the Lyapunov function (16). Consequently, $\dot{W}=0$ at these equilibrium points. If at $t_{0}=0$, the closed-loop system lies to local maximum, it remains in this point for $t>t_{0}$. Nevertheless, if at $t_{0}$ the closed-loop system is away from these equilibrium points, and since $\dot{W}<0$ outside the two equilibrium points, the system state will converge to the equilibrium point $\left(q_{0}=1, \vec{q}=0, \vec{\omega}=0\right)$ and it will remain there for all subsequent time, since in this point $W=\dot{W}=0$. This ends the proof of the almost global asymptotic stability.

Remark 1. From the proof of the Theorem 1, if the closed-loop system is far away from the equilibrium points $\left(q_{0}= \pm 1, \vec{q}=0, \vec{\omega}=0\right)$, the system will approach asymptotically to the point $\left(q_{0}=1, \vec{q}=0, \vec{\omega}=0\right)$, which can be considered an attractor point, whereas $\left(q_{0}=-1, \vec{q}=0, \vec{\omega}=0\right)$ can be considered a repeller point (see Joshi et al. (1995)). However, the repeller point becomes an attractor using the control law $\Gamma_{i}=-\sigma_{\bar{\Gamma}_{i}}\left(\frac{\kappa \omega_{i}}{\rho_{i}}-\kappa q_{i}\right)$ instead of (11). In a practical context, it is essential to select the equilibrium point to be achieved in order to minimize the angular path. Therefore, applying

$$
\Gamma_{i}=-\sigma_{\bar{\Gamma}_{i}}\left(\frac{\kappa \omega_{i}}{\rho_{i}}+\operatorname{sign}\left(q_{0}\right) \kappa q_{i}\right)
$$


ensures that, of the two rotations of angle $\beta$ and $2 \pi-\beta$, the one of smaller angle is chosen. Then, the control law (26) stabilizes globally asymptotically the two-point set in the quaternion space. This can be demonstrated by adapting the previous proof using the following Lyapunov function:

$$
W=\frac{1}{2} \vec{\omega}^{T} J \vec{\omega}+ \begin{cases}2 \kappa\left(1-q_{0}\right), & \text { if } q_{0} \geq 0 \\ 2 \kappa\left(1+q_{0}\right), & \text { if } q_{0}<0\end{cases}
$$

This result is in the spirit of Mayhew et al. (2009).

Remark 2. Note that the stability analysis has been carried out considering the asymptotic condition $q \rightarrow q_{d}=\left[\begin{array}{llll}1 & 0 & 0 & 0\end{array}\right]^{T}$ and with (26) it is possible to achieved $q_{d}=\left[\begin{array}{llll} \pm 1 & 0 & 0 & 0\end{array}\right]^{T}$. In the case where the asymptotic condition $q \rightarrow q_{d}$ with $q_{d} \neq\left[\begin{array}{llll} \pm 1 & 0 & 0 & 0\end{array}\right]^{T}$ is considered, the control law to be applied becomes

$$
\Gamma_{i}=-\sigma_{\bar{\Gamma}_{i}}\left(\frac{\kappa \omega_{i}}{\rho_{i}}+\operatorname{sign}\left(q_{e_{0}}\right) \kappa q_{e_{i}}\right)
$$

where $q_{e}$ represents the attitude error between the current orientation and the desired one.

\subsection{Some properties of the control law}

In practice, the angular velocity is generally obtained by rate gyros. These sensors can measure the angular velocity in a priori specified range depending on the sensors and its technology. The control law (11) allows to take into consideration the angular velocity constraints, i.e. slew rate limits.

Let $\left|\omega_{i_{\max }}\right|$ denote the maximum magnitude angular velocity allowed for the rigid body manoeuvre about the $\mathrm{i}^{\text {th }}$ axis. Let us consider the following state constraint

$$
\omega_{i} \in\left[-\omega_{i_{\max }}, \omega_{i_{\max }}\right]
$$


then, one has the following results.

Corollary 1 (Slew rate constraints). The control law (11) almost globally asymptotically stabilizes the rigid body described by (5) and (6) to the origin. Moreover, setting $\rho_{i}=\left|\omega_{i_{\max }}\right|$ the state constraint (29) is guaranteed to remain satisfied:

- if $\left|\omega_{i}\left(t_{0}\right)\right|>\omega_{i_{\max }}$, then there exists some time $t_{1}>t_{0}$ depending upon the initial conditions such that the state constraint (29) holds for any $t>t_{1}$

- if $\left|\omega_{i}\left(t_{0}\right)\right| \leq \omega_{i_{\max }}$, then the state constraint (29) holds for all $t>t_{0}$

Proof. The claim follows of the proof of Theorem 1 replacing $\rho_{i}$ for $\left|\omega_{i_{\max }}\right|$. First assume that $\left|\omega_{i}\right|>\rho_{i}=\left|\omega_{i_{\max }}\right|$. Then, the control law (11) forces the velocities trajectories to enter to the set $\Psi_{i}=\left\{\omega_{i}:\left|\omega_{i}\right| \leq \rho_{i}=\left|\omega_{i_{\max }}\right|\right\}$ in finite time $t_{1}$ and remain in it thereafter.

In case where $\left|\omega_{i}\left(t_{0}\right)\right| \leq\left|\omega_{i_{\max }}\right|$, from condition $\rho_{i}=\left|\omega_{i_{\max }}\right|$ the angular velocities belong to the set $\Psi_{i}=\left\{\omega_{i}:\left|\omega_{i}\right| \leq \rho_{i}=\left|\omega_{i_{\max }}\right|\right\}$. Thus from Theorem 1, the control law (11) enforces the velocities trajectories to remain in the set $\Psi_{i}$ for all $t>t_{0}$ and the state constraint (29) remains satisfied.

In several situations, the body angular velocities exceed the capability of the rate gyros (for instance, in the case where an external disturbance is present). The following propriety establishes the robustness of the proposed control law towards bounded angular velocities measurements. 


\section{Corollary 2 (Robustness towards bounded angular velocities mea-} surements). Let $\left|\bar{\omega}_{i}\right|>0$ represent the maximum magnitude angular velocity that can be measured by the rate gyro along the $i^{\text {th }}$ axis. Let $\rho_{i}$ be chosen as $\rho_{i}=\left|\bar{\omega}_{i}\right|-\varepsilon$ for some strictly positive $\varepsilon$ sufficiently small. Assume that the angular velocity exceeds the rate gyro limits, that is $\left|\omega_{i}\right|>\left|\bar{\omega}_{i}\right|$, then the control inputs (11) almost globally asymptotically stabilize the rigid body to the origin, in spite of angular velocities measurements saturation.

Proof. Assume that $\left|\omega_{i}\right|>\left|\bar{\omega}_{i}\right|$, setting $\rho_{i}=\left|\bar{\omega}_{i}\right|-\varepsilon$ one has $\left|\omega_{i}\right|>\rho_{i}+\varepsilon$. Consider the candidate Lyapunov function given in (12). With the same reasoning as in the proof of Theorem 1, (15) becomes for some $\varepsilon>0$ sufficiently small.

$$
\dot{V}_{i}=-\frac{\kappa \varepsilon}{\left|\bar{\omega}_{i}\right|}\left|\omega_{i}\right|<0
$$

This establishes the strict decrease of $V_{i}$ when $\left|\omega_{i}\right|>\left|\bar{\omega}_{i}\right|$. Consequently, $\omega_{i}$ enters $\Phi_{i}=\left\{\omega_{i}:\left|\omega_{i}\right| \leq \rho_{i}+\varepsilon=\left|\bar{\omega}_{i}\right|\right\}$ in finite time $t_{1}$ and remains in it thereafter. Hence, the rate gyro is not saturated and the system evolves in the same way that the nominal system (no rate gyro saturation). The same argument is applied to $\dot{V}_{2}$ and $\dot{V}_{3}$.

\subsection{Selection of the feedback gains}

Selecting the feedback gain may be difficult especially when constraints exist between the gains. Here, selecting the gains can be easily done recalling that when the control law is not constrained it becomes:

$$
\Gamma_{i}=-\frac{\kappa}{\rho_{i}} \omega_{i}-\kappa q_{i}
$$

Hence, $\kappa$ tunes how sensible to angles or angular velocities errors the control will be but also how sensible to noise on these variables. This is very 
important within an application context, since the output signal of a low cost rate gyro is very noisy and this can cause instability. $\rho_{i}$ will tune the relative influence between the angular information (through the quaternion) and the velocity information. Note that according to Corollary 1, choosing $\rho_{i}=\left|\omega_{i_{\max }}\right|$ will guarantee the slew rate constraint.

\section{Real-time experimentation to a quadrotor mini-helicopter}

\subsection{Introduction}

In recent years, significant research effort has been directed toward the control, modelling and design of flying robots. This interest arises not only for military purposes but also for civil applications such as disaster monitoring, environmental surveillance or even cinematography. Within flying robots, the quadrotor helicopter has attracted a great interest because of its highly manoeuvrability and the ability to hover. This Vertical Take-Off and Landing (VTOL) vehicle has some advantages over conventional helicopters. Owing to symmetry, this vehicle is simple to design and construct. The small moment of inertia of this aerial robot makes it vulnerable to large angular acceleration. Therefore, for many potential missions an efficient attitude control is crucial. It allows the vehicle to maintain a desired orientation. Several control techniques have been used for the attitude stabilization of the quadrotor mini-helicopter, for instance, PID and LQ control law in Bouabdallah et al. (2004), sliding mode control in Bouabdallah and Siegwart (2005) and Backstepping approach applied in Tayebi and McGilvray (2006). Although the results presented in the above cited works are interesting and founding, the boundedness of the control input is not considered. One partic- 
ular aspect of the quadrotor mini-helicopter dynamics, is that the dynamical model for the attitude may be reduced to that of a rigid body. Therefore, the proposed approach for the stabilization of a rigid body is naturally extended to the attitude stabilization of the quadrotor mini-helicopter. The control attitude strategy presented in the previous section is applied to the attitude regulation of a quadrotor mini-helicopter as the one shown in Fig.1.

\subsection{Quadrotor helicopter dynamics}

The mini helicopter under study has four fixed-pitch rotors mounted at the four ends of a simple cross frame. On this platform, given that the front and rear motors rotate counter-clockwise while the other two rotate clockwise, gyroscopic effects and aerodynamic torques tend to cancel in trimmed flight. The collective input (or throttle input) is the sum of the thrusts of each rotor $\left(f_{1}+f_{2}+f_{3}+f_{4}\right)$. Pitch movement $(\theta)$ is obtained by increasing (reducing) the speed of the rear motor while reducing (increasing) the speed of the front motor. The roll movement $(\phi)$ is obtained similarly using the lateral motors. The yaw movement $(\psi)$ is obtained by increasing (decreasing) the speed of the front and rear motors while decreasing (increasing) the

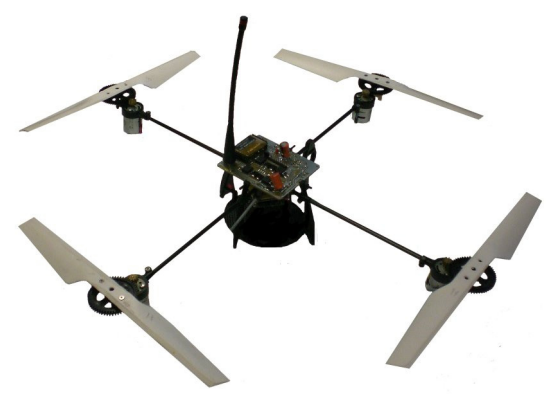

Figure 1: The Quadrotor mini-helicopter prototype of GIPSA-Lab 
speed of the lateral motors. This should be done while keeping the total thrust equal or larger than the helicopter weight in order to cancel the gravity force. In order to model the system dynamics, two frames are defined: the

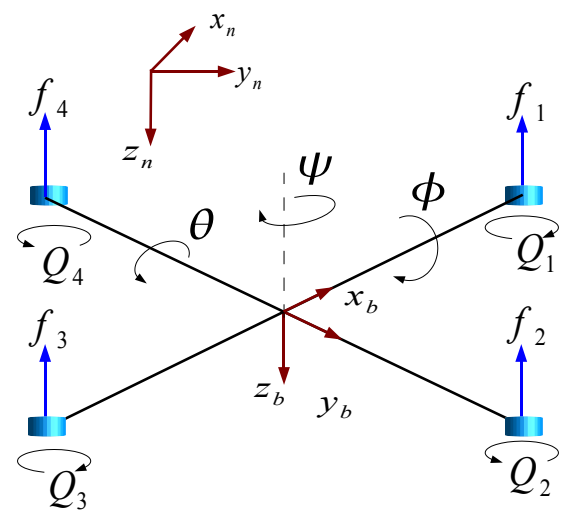

Figure 2: Quadrotor mini-helicopter configuration: the inertial frame $N\left(x_{n}, y_{n}, z_{n}\right)$ and the body-fixed frame $B\left(x_{b}, y_{b}, z_{b}\right)$

inertial frame $N\left(x_{n}, y_{n}, z_{n}\right)$ and the body-fixed frame $B\left(x_{b}, y_{b}, z_{b}\right)$ as shown in Fig.2.

According to Pounds et al. (2002) and section 2, the quadrotor minihelicopter model can be expressed in terms of quaternions

$$
\begin{aligned}
\dot{p} & =v \\
\dot{v} & =\vec{g}^{N}-\frac{1}{m} C^{T}(q) \vec{T} \\
\dot{q} & =\frac{1}{2} \Xi(q) \vec{\omega} \\
J_{h} \dot{\vec{\omega}} & =-\vec{\omega} \times J_{h} \vec{\omega}-\Gamma_{G}+\Gamma
\end{aligned}
$$

where $m$ denotes the mass of the helicopter, $\vec{g}$ is the vector of the gravity

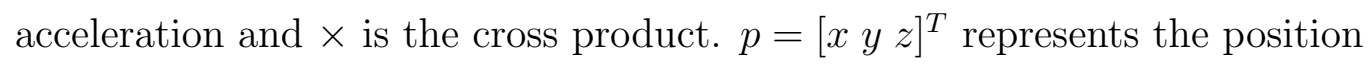


of the origin of the $B$-frame with respect to the $N$-frame, $v=\left[\begin{array}{lll}v_{x} & v_{y} & v_{z}\end{array}\right]^{T}$ is the linear velocity of the origin of the $B$-frame expressed in the $N$-frame, and $\vec{\omega}$ denotes the angular velocity of the helicopter expressed in the $B$ frame. $\Gamma_{G} \in \mathbb{R}^{3}$ contains gyroscopic couples, due to the rotational motion of the mini-helicopter and the four rotors, $\Gamma \in \mathbb{R}^{3}$ is the vector of the control torques and $\vec{T}=\left[\begin{array}{lll}0 & 0 & T\end{array}\right]^{T}$ is the total thrust expressed in the $B$-frame. The attitude model of the four rotor aircraft differs from the general model (5)-(6) in the gyroscopic couples $\Gamma_{G}$. However, it will be proved that the approach of Section 3.1 can still be applied.

Equations (32)-(35) describe the 6 degrees of freedom of the system and can be separated into translational (32)-(33) and rotational (34)-(35) motions.

In this application, the rotors speed may reach high values (more than 200 $\mathrm{rad} / \mathrm{sec})$. Therefore, the reactive couple generated in the free air by rotor $i$ due to rotor drag and the total thrust produced by the four rotors can be respectively approximated by (Kendoul et al. (2005)):

$$
\begin{aligned}
Q_{i} & =k s_{i}^{2} \\
T & =\sum_{i=1}^{4} f_{i}=b \sum_{i=1}^{4} s_{i}^{2}
\end{aligned}
$$

where $s_{i}$ represents the rotational speed of rotor $i . k>0$ and $b>0$ are two parameters depending on the density of air, the radius, the shape, the pitch angle of the blade and other factors (Castillo et al. (2004)).

The vector of gyroscopic couples $\Gamma_{G}$ is given by:

$$
\Gamma_{G}=\sum_{i=1}^{4} J_{r}\left(\vec{\omega} \times \overrightarrow{z_{b}}\right)(-1)^{i+1} s_{i}
$$

where $J_{r}$ is the inertia of the called rotor (composed of the motor rotor itself with the gears). The components of the control torque $\Gamma \in \mathbb{R}^{3}$ generated by 
the rotors are given by $\Gamma=\left[\begin{array}{lll}\Gamma_{1} & \Gamma_{2} & \Gamma_{3}\end{array}\right]^{T}$, with

$$
\begin{aligned}
& \Gamma_{1}=d\left(f_{2}-f_{4}\right)=d b\left(s_{2}^{2}-s_{4}^{2}\right) \\
& \Gamma_{2}=d\left(f_{1}-f_{3}\right)=d b\left(s_{1}^{2}-s_{3}^{2}\right) \\
& \Gamma_{3}=Q_{1}-Q_{2}+Q_{3}-Q_{4}=k\left(s_{1}^{2}-s_{2}^{2}+s_{3}^{2}-s_{4}^{2}\right)
\end{aligned}
$$

where $d$ represents the distance from one rotor to the center of mass of the quadrotor mini-helicopter. Combining (37) with (39)-(41), the torques and forces applied to the helicopter are written in vector form as:

$$
\begin{aligned}
{\left[\begin{array}{c}
\Gamma \\
T
\end{array}\right] } & =\left[\begin{array}{cccc}
0 & d b & 0 & -d b \\
d b & 0 & -d b & 0 \\
k & -k & k & -k \\
b & b & b & b
\end{array}\right]\left[\begin{array}{c}
s_{1}^{2} \\
s_{2}^{2} \\
s_{3}^{2} \\
s_{4}^{2}
\end{array}\right] \\
& =N \bar{s}_{r}
\end{aligned}
$$

with $\bar{s}_{r}=\left[\begin{array}{llll}s_{1}^{2} & s_{2}^{2} & s_{3}^{2} & s_{4}^{2}\end{array}\right]^{T}$ the rotor speeds of the four motors. For our model,

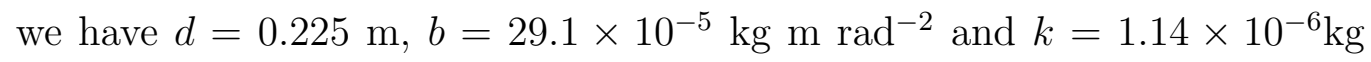
$\mathrm{m}^{2} \mathrm{rad}^{-2}$.

\subsection{Quadrotor torque control design}

In order to stabilize the attitude of the quadrotor mini-helicopter, equations (34)-(35) are used. The rotational motion of the helicopter responds to the control torques arising from the linear combination of the rotational speed of the rotors (42). Hence, the maximum airframe control torque depends on the most higher rotation speed capability of the motors that are used. 
The rotors are driven by DC permanent magnet motors which support a maximum voltage of $9 \mathrm{~V}$ as in Tayebi and McGilvray (2006). When this voltage is applied to the motor the rotation speed reaches $s_{i, \max }=260 \mathrm{rad} / \mathrm{sec}$. Consequently, the maximum torque that is applied to influence the helicopter rotational motion is given by:

$$
\bar{\Gamma}_{1}=0.40 \mathrm{Nm} \quad \bar{\Gamma}_{2}=0.40 \mathrm{Nm} \quad \bar{\Gamma}_{3}=0.15 \mathrm{Nm}
$$

Note that these torques are not identical around the three axis.

In order to avoid undesired damages in the actuators and to maximize its effectiveness, the bounded attitude control presented in the previous section is applied to the subsystem (34)-(35).

Proposition 1. Consider the quadrotor mini-helicopter rotational dynamics described by (34) and (35) with the following bounded control inputs

$$
\begin{aligned}
& \Gamma_{1}=-\sigma_{\bar{\Gamma}_{1}}\left(\frac{\kappa \omega_{1}}{\rho_{1}}+\operatorname{sign}\left(q_{0}\right) \kappa q_{1}\right) \\
& \Gamma_{2}=-\sigma_{\bar{\Gamma}_{2}}\left(\frac{\kappa \omega_{2}}{\rho_{2}}+\operatorname{sign}\left(q_{0}\right) \kappa q_{2}\right) \\
& \Gamma_{3}=-\sigma_{\bar{\Gamma}_{3}}\left(\frac{\kappa \omega_{3}}{\rho_{3}}+\operatorname{sign}\left(q_{0}\right) \kappa q_{3}\right)
\end{aligned}
$$

The parameters $\kappa, \rho_{i}$ with $i \in\{1,2,3\}$ are chosen as in Theorem 1. Then the inputs (44) globally asymptotically stabilize the quadrotor mini-helicopter to the origin, represented by the two-point set in the quaternion space $\left(q_{0}=\right.$ $\pm 1, \vec{q}=0$ and $\vec{\omega}=0$ ).

Proof. The steps of the proof are identical to the proof of Theorem 1 and Remark 1. Indeed, the only difference lies in the vector $\Gamma_{G}$ that adds a term that is canceled because of the relation:

$$
\vec{\omega}^{T} \Gamma_{G}=\vec{\omega}^{T}\left(\vec{\omega} \times \overrightarrow{z_{b}}\right) \sum_{i=1}^{4} J_{r}(-1)^{i+1} s_{i}=0
$$


where $J_{r}$ represents the inertia of the rotor.

Remark 3. While Proposition 1 states that the quadrotor mini-helicopter can be theoretically stabilized from any initial condition, the stabilization depends of the actuator dynamics. A quadrotor with sufficient speed and power could fly a loop without problem. However, hover or flying in straight line upside down it is impossible since the profile of the blades would not allow this to happen.

Remark 4. For the quadrotor mini-helicopter treated in this work, it was observed that the stabilization takes place without a problem if the initial conditions do not exceed the angles $\phi=\theta=40^{\circ}$. For the angle yaw $\psi$ no restrictions exist for the initial condition.

\section{Experimental Results}

The aim of this section is to show the effectiveness of the proposed control. For this, an attitude stabilization in real-time was performed on the quadrotor mini-helicopter prototype of gipsa-lab control system department. This prototype is based in the mechanical structure (airframe, motors and blades) of the Draganflyer III developed by RC Toys. For the elaboration of real-time processing board, it was used an embedded microcontroller $(\mu C)$ C8051F022 manufactured by Silicon Laboratories, an inertial measurement unit (3DM-G from Microstrain), a bi-directional communication unit (SPM2-433-28 from Radiometrix), a power module to drive the motors (MOSFET transistors) and four optical sensors attached to the ends of the mechanical frame to measure the rotor speed. The power is supplied by a 9 Volts Lithium Polymer battery. On the other hand, a communication unit (SPM2-433-28 from 
Radiometrix) is linked to a $\mathrm{PC}$, in order to provide and to obtain the process data. The $\mu C$ acquires the angular velocity and attitude (quaternion) provided by the IMU and it obtains the desired attitude incoming from the ground station. Thus, the $\mu C$ executes the attitude control law and computes the PWM level to control the four motors. Optionally, the embedded system provides the process data to the ground station in order to monitor the experiment. The attitude control loop runs at $f_{s}=76 \mathrm{~Hz}$ due to IMU constraints.

Three experiments have been accomplished. In the first and second experiments the goal is the attitude stabilization at the origin $\left(q_{d}=\left[\begin{array}{llll}1 & 0 & 0 & 0\end{array}\right]\right.$. i.e. $\left.\phi_{d}=\theta_{d}=\psi_{d}=0\right)$. In the third experiment an attitude tracking is performed. The desired thrust is $T \geq m g=4.59 \mathrm{~N}$ in order to compensate the weight of the quadrotor during the experiment. The quadrotor inertia matrix is $J_{h}=\operatorname{diag}(8.28,8.28,15.7) \times 10^{-3}$. Furthermore, from motors characteristics the maximum torque frame that can be applied is $\bar{\Gamma}=\left[\begin{array}{llll}0.40 & 0.40 & 0.15\end{array}\right]^{T} \mathrm{Nm}$. The tuning parameters of the control input are selected according to the discussion in section 3.3. The maximum angular velocity allowed in the experiment is fixed to $\left|\omega_{1,2_{\text {max }}}\right|=4.2 \mathrm{rad} / \mathrm{sec}\left(240^{\circ} / \mathrm{sec}\right)$ and $\left|\omega_{3_{\max }}\right|=1.74$ $\mathrm{rad} / \mathrm{sec}\left(100^{\circ} / \mathrm{sec}\right)$, then, $\rho_{1,2}=4.2$ and $\rho_{3}=1.74$. According to Theorem 1 , $\kappa=0.075$.

\subsection{Stabilization}

In the first experiment the control capabilities are tested to stabilize the system to the origin. The obtained results are depicted in Fig.3 - Fig.5. According to the angles trajectories, the stabilization is achieved in $1.5 \mathrm{sec}$, which is a very suitable time. Moreover, the constraints in the control signal 
are largely satisfied.

\subsection{Disturbance rejection}

In the second experiment, the robustness of the proposed controller towards disturbance rejection has been tested. The disturbances along each axis (in both directions) are introduced into the system after to achieve the attitude stabilization. As can be seen in the Fig.6 and Fig.7, the disturbance produces a large error in the yaw angle as well as in angular velocity $\omega_{z}$. Consequently, the control signal $\Gamma_{3}$ reaches its limit $( \pm 0.15 N \cdot m)$ (see Fig. 8) and takes action on the system to overcome the disturbances. A similar rejection disturbance is observed for the pitch and the roll angle, while only feasible control signals are applied to the system. This study case shows that the controller proposed in this paper is robust towards external disturbances. The control law maximizes the effectiveness of the actuators without endangering the system stability. This robustness property is essential in real missions where aerodynamic forces and others factors are present.

\subsection{Attitude tracking}

The third experiment deals with the attitude tracking. It is observed (see Fig. 9) that the controller is able to follow the reference signal $r_{t}=$

$9^{\circ}+9^{\circ} \sin \left(\frac{\pi}{15} t\right)$ along the axis $z_{b}$. This experiment shows the performance of the proposed control scheme to track a smooth slow reference attitude, even though the control law is designed for stabilization. 


\section{Conclusions}

In this paper, a new bounded control law for the global stabilization of a rigid body is proposed. The objective of this control approach is to maximize the effectiveness of the actuators without taking a risk in the system stability. The control design takes into account the slew rate limits, avoiding possible rate gyro saturations. The presented scheme control is simple. It is based on nested saturation approach and the attitude is parameterized by the unit quaternion. The proposed approach is applied to the stabilization of a quadrotor mini-helicopter. The implementation in real-time is achieved. Several experiments are performed showing the performance in terms of settling time, disturbance rejection and trajectory tracking. Still to compare the proposed approach with other control schemes. However, owing to sim-

plicity, the proposed control law is suitable for application where on-board computational resources are limited.

H. Bang, M.J. Tahk, and H.D. Choi. Large angle attitude control of spacecraft with actuator saturation. Control engineering practice, 11(9):989997, 2003. ISSN 0967-0661.

C. Belta. On controlling aircraft and underwater vehicles. In IEEE International Conference on Robotics and Automation, 2004.

S.P. Bhat and D.S. Bernstein. A topological obstruction to global asymptotic stabilization of rotational motion and the unwinding phenomenon. In American Control Conference, 1998.

J. D. Boskovic, S.-M. Li, and R. K. Mehra. Robust stabilization of spacecraft 
in the presence of control input saturation using sliding mode control. In AIAA Guidance, Navigation, and Control Conference and Exhibit, 1999.

S. Bouabdallah and R. Siegwart. Backstepping and sliding-mode techniques applied to an indoor micro quadrotor. In IEEE International Conference on Robotics and Automation, 2005.

S. Bouabdallah, A. Noth, and R. Siegwart. PID vs LQ control techniques applied to an indoor micro quadrotor. In International Conference on Intelligent Robots and Systems, 2004.

P. Castillo, A. Dzul, and R. Lozano. Real-time stabilization and tracking of a four-rotor mini rotorcraft. IEEE Transactions on Control Sytems Technology, 12(4):510-516, 2004.

J. C. K. Chou. Quaternion kinematic and dynamic differential equations. IEEE Transactions on Robotics and Automation, 8(1):53-64, 1992.

O. Egeland and J.-M. Godhavn. Passitvity-based adaptive attitude control of a rigid spaceraft. IEEE Transactions on Automatic Control, 39(5):842846, 1994.

O. Fjellstad and T. Fossen. Quaternion feedback regulation of underwater vehicles. In 3rd IEEE Conference on Control Application, 1994.

O. Hegrenas, J. T. Gravdahl, and P. Tondel. Attitude control by means of explicit model predictive control, via multi-parametric quadratic programming. In American Control Conference, 2005. 
S. M. Joshi, A. G. Kelkar, and J. T. Wen. Robust attitude stabilization of spacecraft using nonlinear quaternion feedback. IEEE Transactions on Automatic Control, 40(10):1800-1803, 1995.

F. Kendoul, I. Fantoni, and R. Lozano. Modeling and control of a small aircraft having two tilting rotors. In $44^{\text {th }}$ IEEE Conference on Decision and Control and European Control Conference, 2005.

D. Koditschek. Application of a new lyapunov function to global adaptive attitude tracking. In Proceedings of the 27th Conference on Decision and Control, 1988.

R. Kristiansen and P. J. Nicklasson. Satellite attitude control by quaternionbased backstepping. In American Control Conference, 2005.

M. Krstić and P. Tsiotras. Inverse optimal stabilization of a rigid spaceraft. IEEE Transactions on Automatic Control, 44(5):1042-1049, 1999.

C. Mayhew, R. Sanfelice, and A. Teel. Robust global asymptotic attitude stabilization of a rigid body by quaternion-based hybrid feedback. In Joint 48th IEEE Conference on Decision and Control and 28th Chinese Control Conference, 2009.

M. Osipchuck, K. D. Bharadwaj, and K. D. Mease. Achieving good performance in global attitude stabilization. In American Control Conference, 1997.

P. Pounds, R. Mahony, P. Hynes, and J. Roberts. Design of a four-rotor aerial robot. In Australian Conference on Robotics and Automation, 2002. 
S. L. Scrivener and R. C. Thompson. Survey of time-optimal attitude maneuvers. Journal of Guidance, Control and Dynamics, 17(2):225-233, 1994.

M. D. Shuster. A survey of attitude representations. Journal of the astronautical sciences, 41(4):439-517, 1993.

S. N. Singh and W. Yim. Nonlinear adaptive backstepping design for spacecraft attitude control using solar radiation pressure. In $41^{\text {st }}$ IEEE conference on Decision and Control, 2002.

C Song, S.-J. Kim, S.-H. Kim, and H. S. Nam. Robust control of the missile attitude based on quaternion feedback. Control Engineering Practice, 14: 811-818, 2005.

A. Tayebi. Unit quaternion-based output feedback for the attitude tracking problem. IEEE Transactions on Automatic Control, 53(6):1516-1520, 2008.

A. Tayebi and S. McGilvray. Attitude stabilization of a vtol quadrotor aircraft. IEEE Transactions on Control Systems Technology, 14(3):562-571, 2006.

A.R. Teel. Global stabilization and restricted tracking for multiple integrators with bounded controls. Systems \& Control Letters, 18:165-171, 1992.

P. Tsiotras. New control laws for the attitude stabilization of rigid bodies. In $13^{\text {th }}$ IFAC Symposium on Automatic Control in Aerospace, 1994.

P. Tsiotras and J. Luo. Control of underactuated spacecraft with bounded inputs. Automatica, 36(8):1153-1169, 2000. 
J. T. Wen, S. Seereeram, and D. S. Bayard. Nonlinear predictive control applied to spacecraft attitude control. In American Control Conference, 1997.

J.T. Wen and K. Kreutz-Delgado. The attitude control problem. IEEE Transactions on Automatic Control, 36(11):1148-1162, 1991.

B. Wie and J. Lu. Feedback control logic for spacecraft eigenaxis rotations under slew rate and control constraints. Journal of Guidance, Control and Dynamics, 18(6):1372-1379, 1995.

B. Wie, H. Weiss, and A. Arapostathis. Quaternion feedback regulator for spacecraft eigenaxis rotations. Journal of Guidance, Control and Dynamics, 12(3):375-380, 1989.

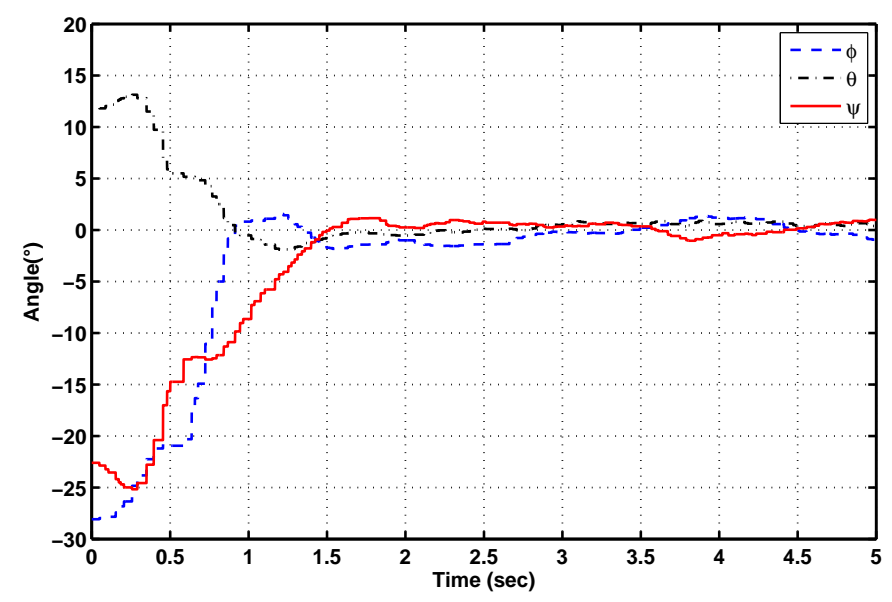

Figure 3: Stabilization: The convergence of the roll, pitch and yaw angles with initial conditions $\phi=-28^{\circ}, \quad \theta=13^{\circ}, \quad \psi=-23^{\circ}$. 


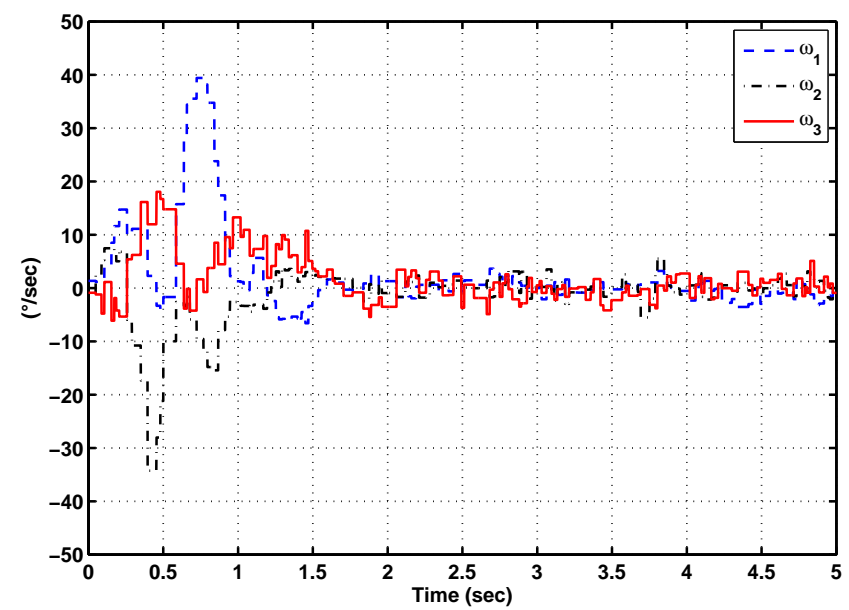

Figure 4: Stabilization: The evolution of the angular velocity.

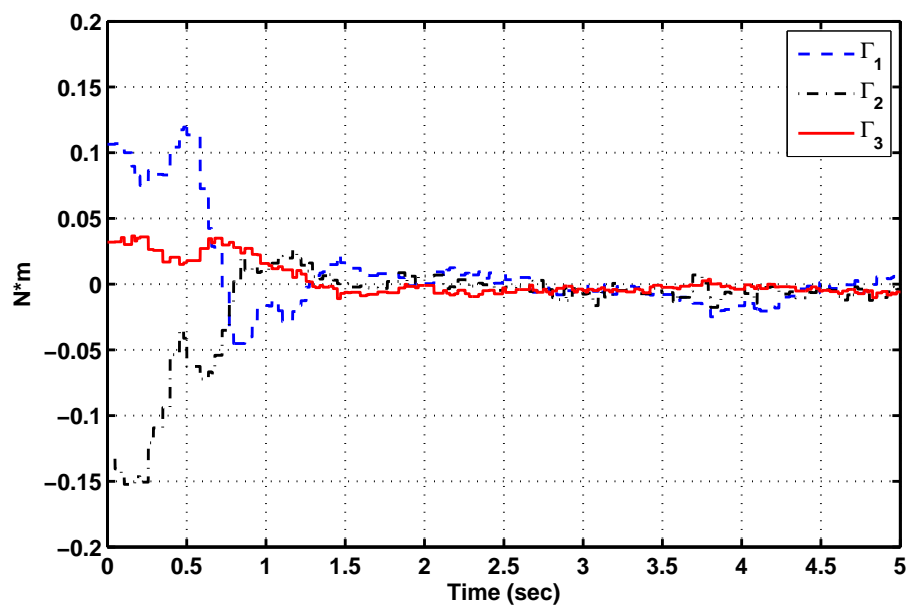

Figure 5: Stabilization: The bounded control torque. 


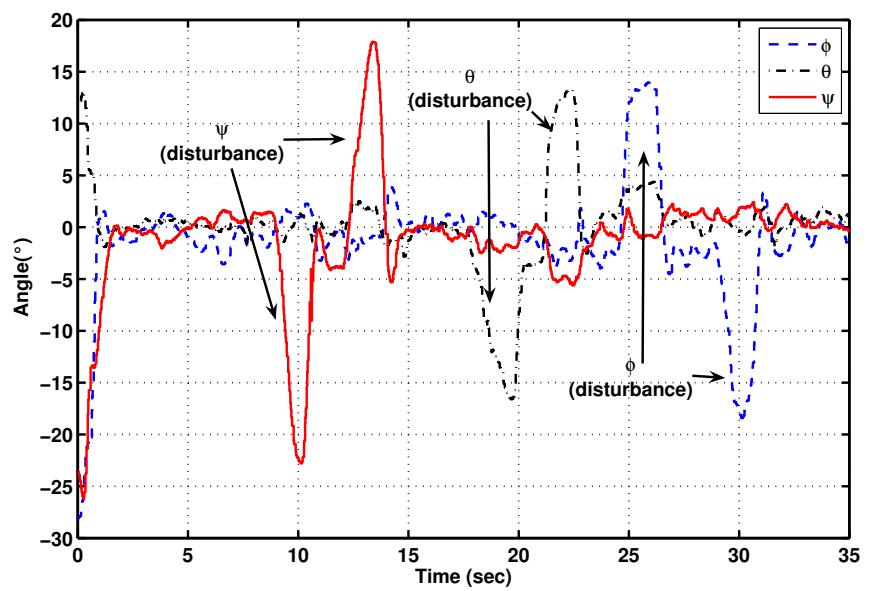

Figure 6: Disturbance rejection: The convergence of the roll, pitch and yaw angles, while the system is subject to external disturbances.

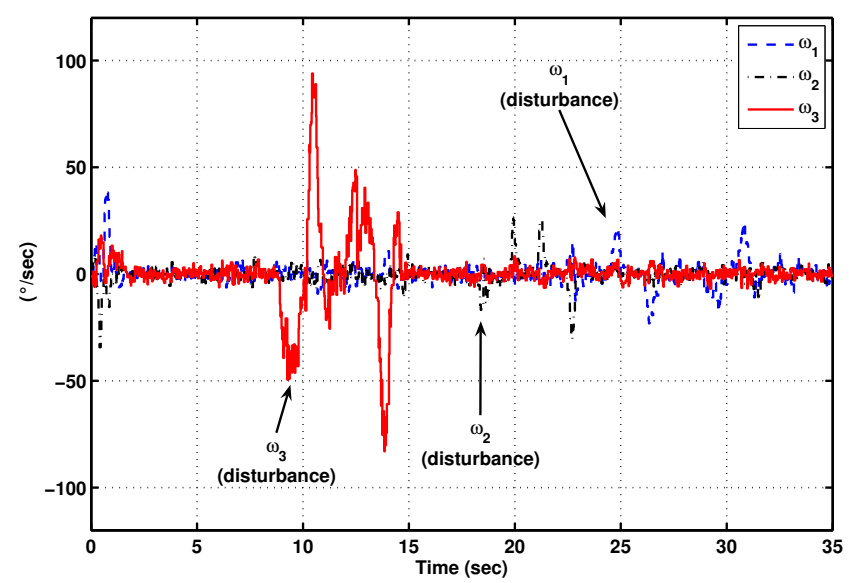

Figure 7: Disturbance rejection: The evolution of the angular velocity, while the system is subject to external disturbances. 


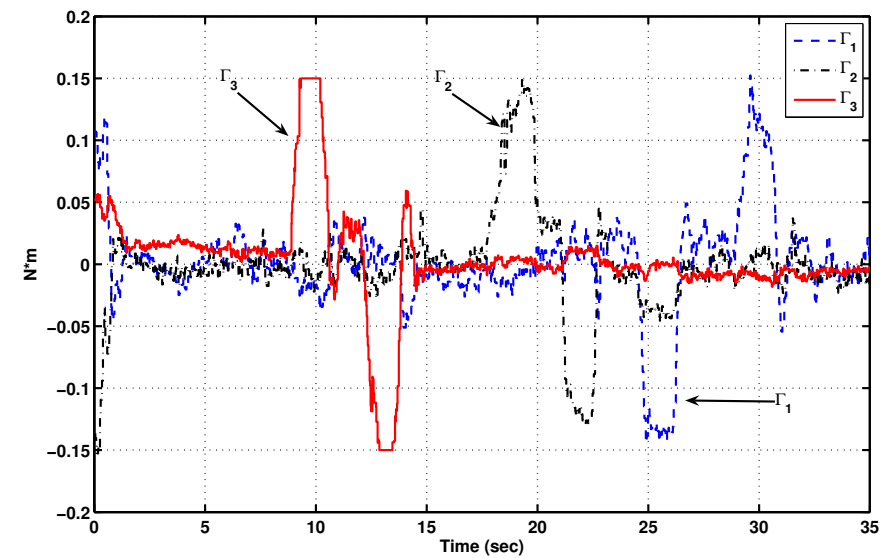

Figure 8: Disturbance rejection: The bounded control torque signal, while the system is subject to external disturbances. The control constraints are satisfied.

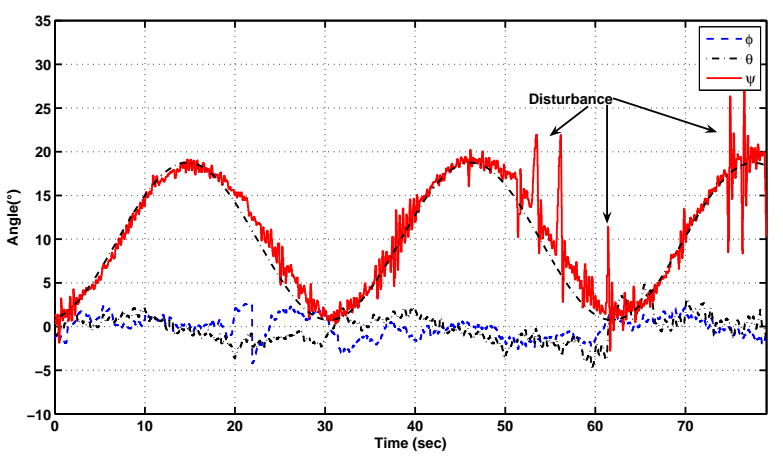

Figure 9: Tracking: The evolution of the roll, pitch and yaw angles, while the system tracks a sinus signal along the axis $z_{b}$. 


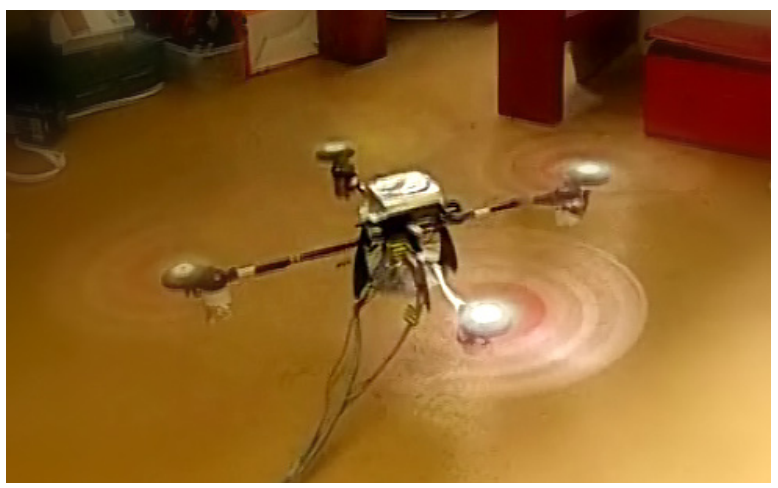

Figure 10: The quadrotor mini-helicopter in flight. 